\title{
Mapping mammalian synaptic connectivity
}

\author{
Chaehyun Yook · Shaul Druckmann · Jinhyun Kim
}

Received: 27 February 2013 / Revised: 17 June 2013 / Accepted: 24 June 2013 / Published online: 18 July 2013

(C) The Author(s) 2013. This article is published with open access at Springerlink.com

\begin{abstract}
Mapping mammalian synaptic connectivity has long been an important goal of neuroscientists since it is considered crucial for explaining human perception and behavior. Yet, despite enormous efforts, the overwhelming complexity of the neural circuitry and the lack of appropriate techniques to unravel it have limited the success of efforts to map connectivity. However, recent technological advances designed to overcome the limitations of conventional methods for connectivity mapping may bring about a turning point. Here, we address the promises and pitfalls of these new mapping technologies.
\end{abstract}

Keywords Synaptic connectivity · Brainbow $\cdot$ Array tomography $\cdot$ mGRASP $\cdot$ Trans-synaptic tracer .

Neurological disorders

\section{Introduction}

More than a century ago, the visionary Spanish neuroanatomist Santiago Ramón Cajal (Nobel Laureate 1906) proposed what is often called the "neuron-doctrine," the idea that neurons are the structural and functional units of the brain, and guided by this idea, proceeded to explore the

C. Yook · J. Kim $(\bowtie)$

Center for Functional Connectomics (CFC), L7-7205,

Korea Institute of Science and Technology (KIST), 39-1

Hawolgokdong, Seongbukgu, Seoul 136-791, Korea

e-mail: kimj@kist.re.kr

C. Yook

Department of Biological Science, KAIST, Daejeon, Korea

S. Druckmann

Howard Hugh Medical Institute, Janelia Farm Research Campus,

Ashburn, USA complex architecture of neuronal networks [1, 2]. Neurons in networks communicate with one another through a special bridge-like structure called a synapse. Neuronal connections were traditionally determined by electrophysiological measurement from linked pairs of cells to determine the strength as well as the existence of a synapse, yet this approach has a very low throughput [3]. To this day, neuroscientists continue to seek new high-throughput ways to investigate neuronal circuits by mapping synaptic connectivity [4-10]. Recently, in 2005, the terms "connectome" and "connectomics" were coined and have since been widely used to describe this effort [11] ("-ome" as an analogy to "genome" is taken to signify complete maps of connections in a brain or a brain area). In 2009, the Human Connectome Project was launched by the National Institutes of Health (http://www.humanconnectomeproject. org) with the goal of building macro-scale descriptions of structural and functional connectivity in healthy human brains (strictly speaking, it would have been better named the "projectome" since the methods used in the Human Connectome Project can provide only macro-level profiles of nerve bundle projections); these profiles can be used to predict the probability of connectivity but not specific connections between given cells $[9,12,13]$, and, increasingly, scientific endeavors are underway to map synaptic connectivity in other organisms (e.g., nematode, fruit fly, and mouse) at multiple scales: micro-scale (nano- or micrometer resolution) for synapse-by-synapse or neuron-by-neuron connectivity, meso-scale for local circuits, and macro-scale (millimeter resolution) for entire brains [4, 14, 15].

However, there are fundamental challenges to reconstructing synaptic connections. First, the synapse is a nanometer-scale structure located along neuronal processes that are very fine (less than a micrometer in diameter), but also very long (sometimes more than a few millimeters 
in length). Accordingly, synaptic reconstruction requires both high-resolution and large-scale mapping, which are difficult to achieve together. Second, in many areas, neurons with interdigitating processes are packed very densely in networks, so that disambiguating individual neurons is time- and labor-intensive. Given these challenges, as was also the case for the Human Genome Project, skeptical views on the connectomics project have emerged (the great brain mapping debate at Columbia University, 2012) [16]. A cautionary perspective is provided by the only organism whose entire connectome and genome have been mapped, the nematode $C$. elegans, which has 302 neurons and 97 megabases (compare with 86-100 billion neurons and 300 megabases in the human) $[14,17]$. Some neuroscientists point out that we still do not understand how some of the most basic behaviors of this tiny creature are governed despite its simplicity, and suggest that systematic connectivity mapping will not provide efficient ways to answer questions about brain function.

Yet, most neuroscientists agree that furthering knowledge of synaptic connections and extracting the principles of these connections will aid in understanding how the brain works $[8,18,19]$. Some skeptics focus on the cost-gain economics of the enterprise since the capacity of currently available techniques for connectivity mapping indeed forms a bottleneck. Therefore, developing and improving new technologies for synaptic mapping will be essential at each step of the process: neuronal labeling, imaging, and reconstruction. In Cajal's time, the valuable staining method developed by Camillo Golgi (Nobel Laureate 1906) in 1873, an up-to-date Zeiss microscope, and his talent for drawing allowed him to discover many important aspects of the organization of the nervous system [1, 2, 20, 21]. In modern times, the discovery of fluorescent tags, advanced genetic engineering for labeling, innovations in optics for imaging, and tremendous increases in computer power for reconstructing all facilitate connectomics. Here, we review advanced techniques for mapping connectivity, their promise and pitfalls, with particular attention to visualizing connectivity.

I. Electron-based imaging approach

II. Photon-based imaging approach

\section{II-1. Brainbow}

II-2. Array Tomography

II-3. mGRASP

II-4. Trans-synaptic tracing

II-5. New LM: Super-resolution LM and SPIM with transparent brain

III. Functional connectivity

IV. Synaptic connections in neurological disorders

V. Interactions with computational modeling

\section{Electron-based imaging approach}

Electron microscopy (EM) developed by the German physicist Ernst Ruska (Nobel Laureate 1986) in 1931 provides much better lateral resolution (approximately 50-pm resolution) than light microscopy (LM), which is limited by diffraction ( 200-nm resolution). Crucially, resolving the $\sim 20$-nm-wide synaptic cleft of a densely packed synapse is beyond the resolution of typical LM but is well within that of EM. Accordingly, EM-based approaches have been considered the option of choice for complete synaptic reconstruction. While a sparsemapping approach allowed Cajal to propose his connection diagrams using information only about a few Golgilabeled neurons at a time, EM-based dense-mapping now provides a relatively complete picture of neuronal structure [14]. EM dense reconstruction allows achievements that are almost impossible to achieve by LM-based mapping, yet the process of synaptic mapping through $\mathrm{EM}$, especially data acquisition and reconstruction, is extremely time- and labor-intensive. Therefore, much effort has been devoted to improving the throughput of the EM process. To improve data acquisition, several new approaches have been developed (Fig. 1A). Serial block-face electron microscopy (SBFEM) is designed to obtain well-aligned images by serially imaging backscattered electrons from the surface of tissue embedded in a sample block, then slicing that surface away, and then imaging the newly revealed surface. The sample block is sliced by either a diamond knife [22] or focused ion beam (FIB) [23] incorporated into the EM chamber. With a diamond knife, SBFEM has been reported to have voxel resolution of $20 \times 20 \times 25 \mathrm{~nm}$ [4, 24]. The lateral resolution of scanning electron microscopy depends on field-emitted electron density; its $z$-resolution can be improved by cutting thinner slices, and, in case of SBFEM, by using lower electron beam energy to limit penetration depth [22]. Serial section scanning electron microscopy (SSSEM) combined with a sample collection system (automatic tape-collection lathe ultra microtome, ATLUM) offers voxel resolution as high as $4 \times 4 \times 50 \mathrm{~nm}$ as well as reliable section collection with reduced section distortion [24]. Both systems have sufficient resolution to trace dense neuropils and to reconstruct the synaptic connectivity of neural circuits. For the resolution needed to observe even gap junctions, automated transmission EM (ATEM) offers a combination of higher-resolution serial TEM with automated image alignment and registration [25]. However, putting aside that EM-based imaging processes take relatively more time than LM-based processes, it is the analysis of these images and the reconstruction of neurons and their connections that really hold back the progress of EM-based 


\section{A EM-based approaches}

a. SBFEM

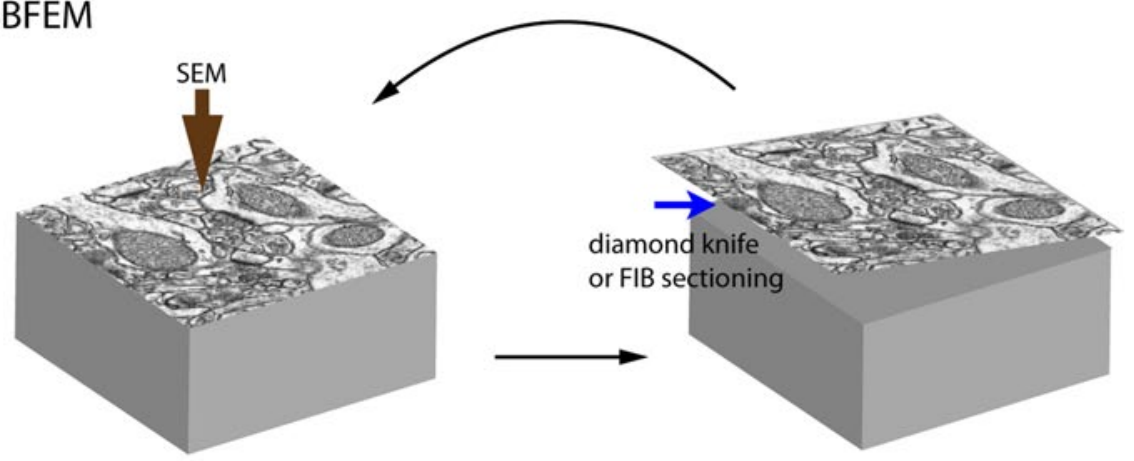

b. SSSEM with ATLUM

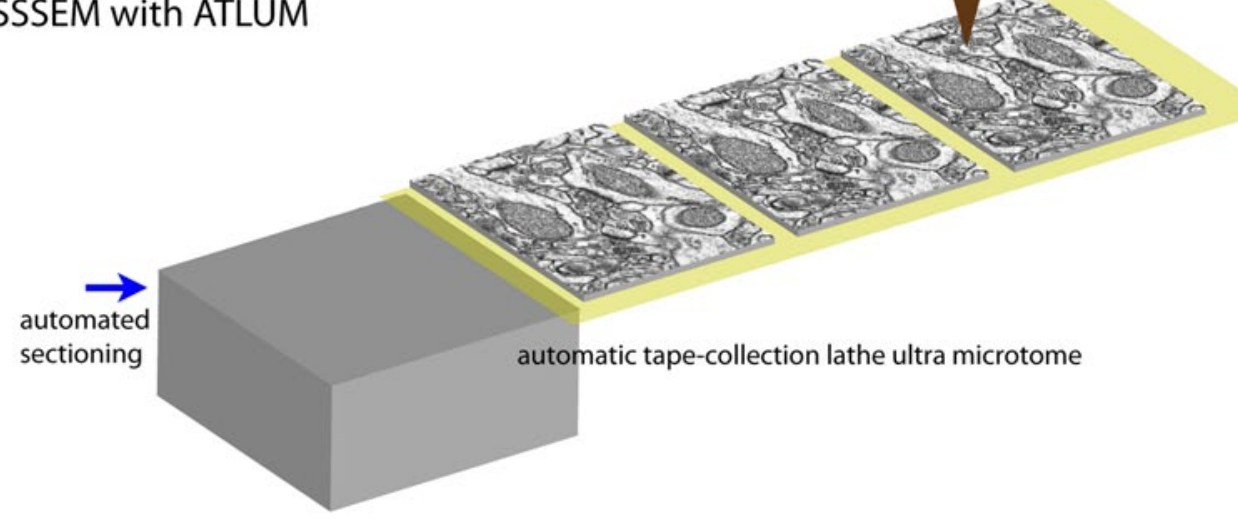

\section{B confocal vs. fSPIM}
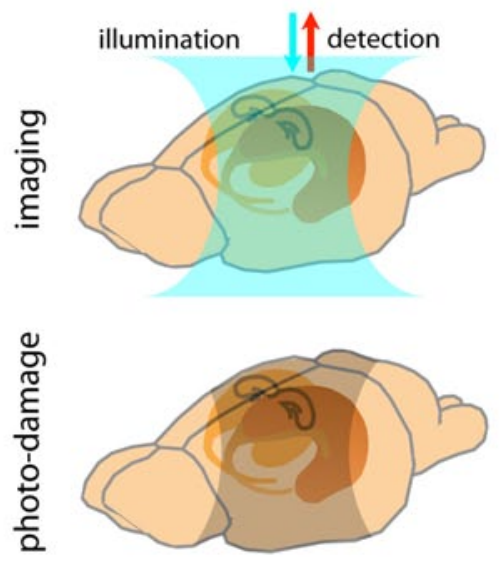

conventional confocal microscopy

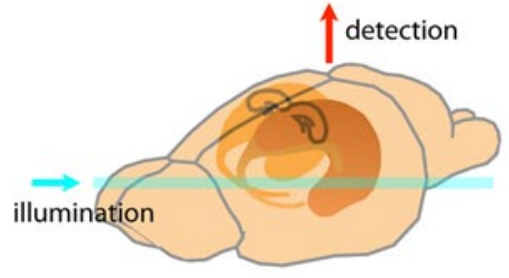

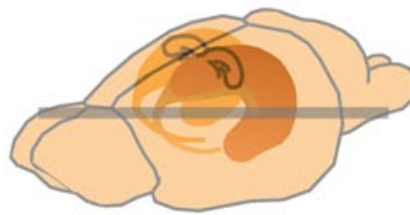

light sheet microscopy

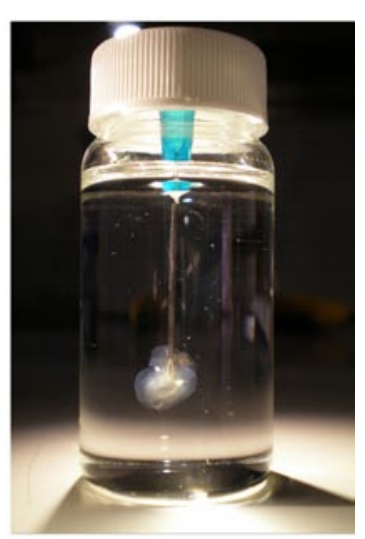

cleared mouse brain

Fig. 1 EM approaches and new LM (fSPIM)

connectivity mapping. Since an EM image contains every visible detail within its field of view, selecting relevant information, such as distinguishing contours of interest from irrelevant ones, takes much more time than would be the case for a sparse-labeled LM image (200-400 h/ $\mathrm{mm}$ for manually tracing SBFSEM images versus 0.25 $1 \mathrm{~h} / \mathrm{mm}$ for single neuron-labeled LM images) [24, 26, 27]. To expedite this intensively time-consuming step, 
efforts to develop a reliable computer-based tracing method, such as through machine-learning algorithms, are underway [28, 29]. However, thus far, unfortunately, even after important new advances in EM-based methodology, reconstructing neuronal tissue remains a relatively time-consuming and volume-limited endeavor.

\section{Photon-based imaging approaches}

Recently, more sophisticated genetic and optical methods have been advanced to circumvent the low resolution of photon-based strategies for mapping synaptic connectivity (Fig. 2).

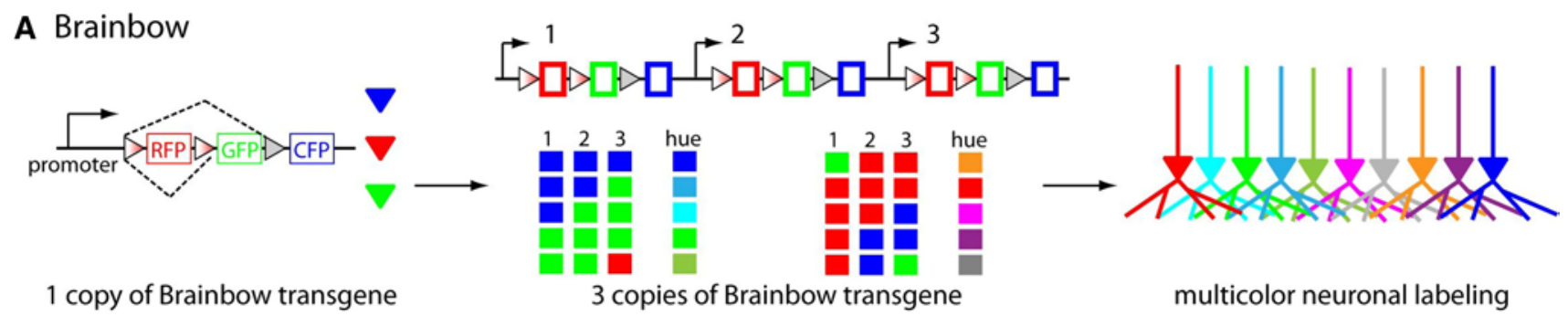

B Array Tomography

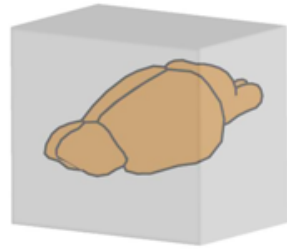

embeded specimen

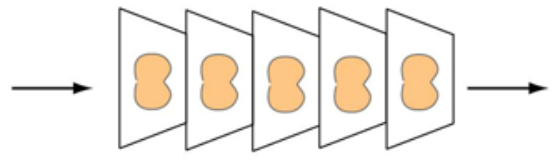

ultrathin serial sections in array

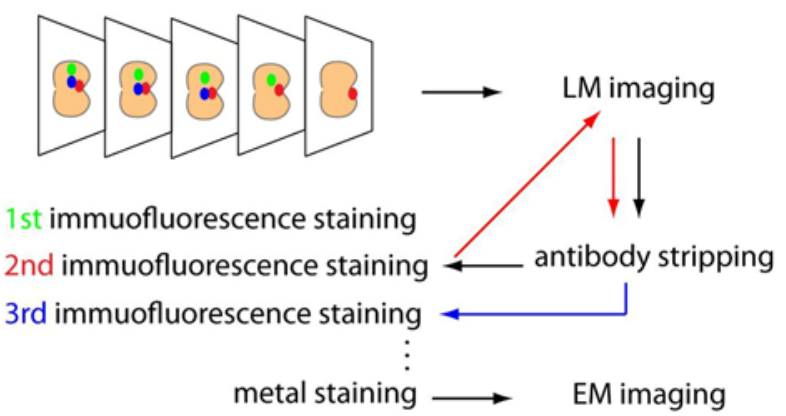

C MGRASP

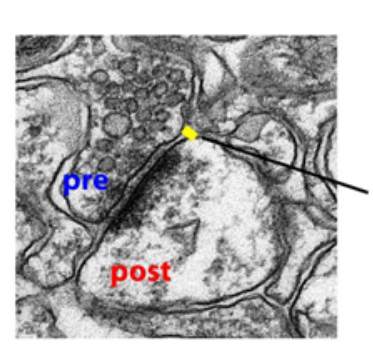

synapse in EM

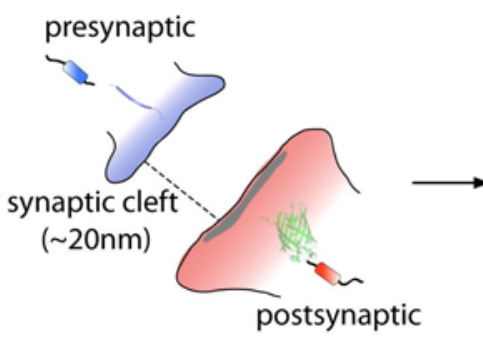

synaptically targeted mGRASP presynaptic

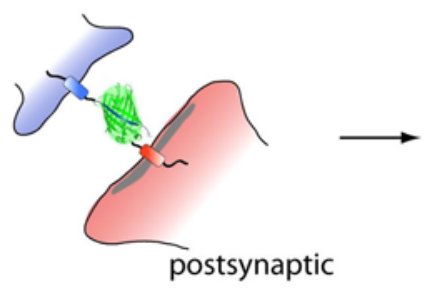

resconstitution of mGRASP

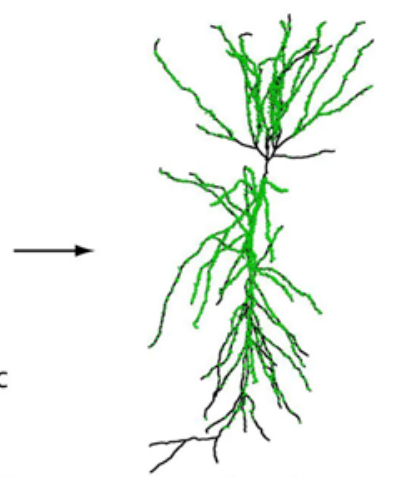

reconstruction of synapses

\section{D trans-synaptic rabies virus}

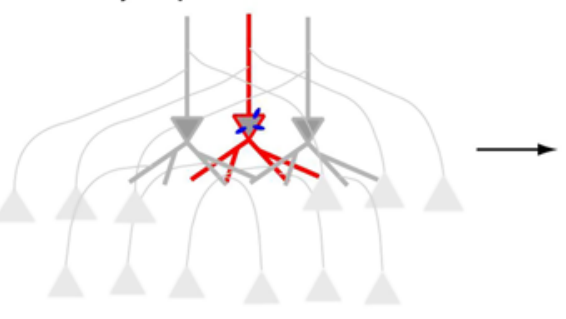

transfection of TVA+RG+RFP

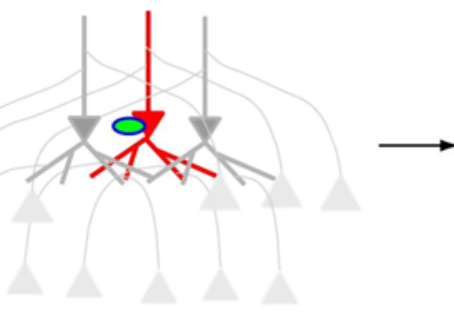

infection of $\Delta G, E n v A-G F P$

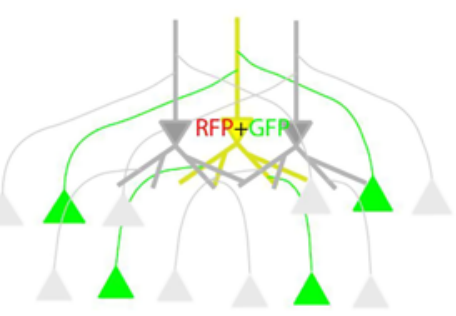

mono-synaptic retrograde tracing

Fig. 2 Principal of LM approaches for mapping mammalian synaptic connectivity 


\section{Brainbow}

Historically, the degree of overlap between the axonal arbor and the dendritic arbor of sparsely labeled neurons, as measured by LM, has been used to infer the presence of synaptic connectivity [8]. However, unless the labeling is very sparse, it is difficult to reconstruct the processes of individual neurons. To disambiguate each neural process from densely packed axons and dendrites, Lichtman and his group [7, 30, 31] set out to label thousands of neurons with random colors drawn from a set originally consisting of membrane-permeable fluorescent dyes, and later of fluorescent proteins. Genetic labeling can be achieved through an elegant approach using genetic switches and multi-copied transgene integration, called Brainbow [31]. Brainbow is based on the combinatorial and stochastic expression of concatenated multiple fluorescent proteins (collectively referred to as XFPs), in which each spectrally distinct XFP is floxed with variants of the loxP site. Multiple copies of this transgene are inserted into the mouse genome. Stochastic recombination events mediated by Cre recombinase determine which combination of XFPs from the multiple copies of transgene will be formed. The many possible combinations of XFP transgenes result in many unique colors for different neurons. For example, if there are three transgene copies in a Brainbow mouse line, each containing three spectrally distinct XFPs, a neuron can have one of ten hues. Neurons labeled with different colors can be distinguished from one another much more easily. Accordingly, by separating the different color channels, Brainbow allows neurons to be reconstructed one-by-one even within a dense population, thereby extending information obtained by LM-based tracing to the level of the neuronal population. In other words, Brainbow permits the challenging dense reconstruction problem to be addressed by solving the much simpler sparse neuron reconstruction problem. In addition, Brainbow greatly simplifies projection pattern reconstruction since the unique color of each neuron remains recognizable along the projection, and therefore usually there is no need to trace the projection all along its path. This results in significant savings in time when tracing long-range circuits. Lastly, Brainbow can assist timelapse imaging or developmental studies since the unique color profile of each neuron remains stable. Very recently, a new second generation of Brainbow has been developed that uses better detectable and antigenically distinct fluorescent proteins or flp recombinase-FRT (Flpbow) [32]. On the negative side, although Brainbow allows identification of neuronal processes by differentially coloring individual neurons, and allows their synapses to be inferred from neurite contacts, the diffraction-limited resolution of LM is insufficient to unambiguously confirm the presence of actual synapses. This inference technique thus appears to be useful for only a subset of synaptic connections [33, 34]. Thus, additional technologies are usually needed to identify synapses.

\section{Array tomography}

Array tomography (AT) combines LM and EM approaches to resolve synapses by using multiple antibodies to label synaptic markers $[35,36]$. It benefits from the high throughput of LM, high z-resolution of EM, and improved quantitative reliability of information obtained through multi-immunofluorescence. To use this method, a mouse brain is embedded in hydrophilic resin and is then serially sectioned into 50-200-nm ultrathin slices. Then, a long array of these serial sections is repeatedly stained for immunofluorescence by a large number of multiplex markers. Because samples prepared for AT are also compatible with EM, AT can combine LM and EM images. Ultrathin physical sectioning overcomes some obstacles typically presented by immunofluorescence staining and imaging approaches: it permits depth-independent immunostaining and imaging and improves upon the $z$-axis resolution of conventional confocal microscopy $(\sim 700 \mathrm{~nm})$. The protein components of individual synapses are revealed by large sets of markers labeled through repeated cycles of antibody stripping and re-staining. This process, called a single-synapse analysis or a synaptogram, offers insights into synapse molecular diversity [36]. In addition, automated steps for imaging and aligning serial sections allow the reconstruction of neural circuits in large volumes of tissue. Thus, AT reveals not only anatomical circuits but also the synaptic proteome. These combined benefits are a unique advantage. However, a potential disadvantage is that AT relies entirely on antibody staining, leading to concerns that some methods for preparing brain tissue can sharply decreases its antigenicity, and that the fidelity of costly antibodies can vary significantly, potentially resulting in ambiguous and incomplete results.

\section{mGRASP}

Another new genetically controlled method to resolve synapses at the level of LM, termed GFP reconstitution across synaptic partners (GRASP), is synapse-specific labeling with two complementary GFP components [37, 38]. GRASP is based on two non-fluorescent split-GFP fragments (called spGFP1-10 and spGFP11) tethered to synaptic membranes in each of two neuronal populations. When two neurons, each expressing one of the fragments, are tightly opposed across a synaptic cleft, fluorescent GFP is reconstituted. More recent mammalian GRASP (mGRASP) techniques can precisely label actual synapses, not nonsynaptic membrane contacts, by engineering spGFP 
carriers that are specifically targeted to synaptic membranes and that accommodate the physical spacing of the synaptic cleft [38]. The manifest benefit of mGRASP technology is the accurate nanometer-scale $(\sim 20 \mathrm{~nm})$ detection of synapses while circumventing the diffraction limitations of LM. When tested with known synaptic and non-synaptic connections in samples full of axonal contacts, mGRASP has been shown to specifically detect actual synapses with no or few false positives. When combined with specialized analysis software [38, 39], mGRASP can relatively quickly reveal the precise locations and numbers of synapses along postsynaptic dendrites, sites responsible for determining many important characteristics of signal processing. Thus, mGRASP technology is suitable for imaging large-scale connectivity patterns. A potential concern, though, is that this technique sometimes registers false negatives, making it difficult to determine absolute numbers of synapses. The problem of false negatives is common to all LM approaches and varies with instrumental parameters (i.e., laser power, emission spectra, etc.). Further optimization of mGRASP technology and applying it in combination with other technologies will lead to useful new tools for mapping mammalian synaptic connectivity.

\section{Trans-synaptic tracing by rabies virus}

Another method using LM is based on the anterograde and retrograde label of neuronal circuits by trans-synaptic tracers. Among retrograde viral trans-synaptic tracers, the modified rabies virus system seems to be the most promising for mapping synaptic connectivity [40-42]. The rabies virus is a negative-sense, single-stranded RNA virus enveloped by rabies glycoprotein (RG) $[43,44]$ that travels retrogradely between synaptically connected neurons. Interactions between the RG and its receptor in host cells are crucial for the initial and the subsequent trans-synaptic infections. The receptor for RG appears to be restricted to presynaptic nerve terminals [45], possibly explaining the highly selective retrograde trans-synaptic spread of rabies [46-48]. The synapse specificity of rabies virus spread has been assessed anatomically and electrophysiologically in the context of well-characterized neuronal connections $[49,50]$. Wild-type rabies may make multi-synaptic jumps, making it difficult to determine which of the labeled neurons is directly connected to which other neuron. However, recently developed genetic modifications, such as the deletion of an essential envelope glycoprotein $(\Delta G)$ that is trans-complemented in only specific cells, allows monosynaptic retrograde tracing $[41,42,51]$. Specifically, $\Delta \mathrm{G}-$ rabies virus packaged with the avian virus envelope protein EnvA, will only infect cells expressing avian virus receptor TVA, one not expressed in mammalian cells [52]. Delivery of TVA along with RG into a specific set of neurons followed by $\Delta$ G-rabies virus expressing GFP, for example, allows visualizing synaptically connected pairs of neurons. Specific expression of TVA and RG is achieved by using cell-specific Cre mouse lines or by single-cell electroporation in vivo. Further engineering of the rabies virus-based system, for instance, to generate a modified version with lymphocytic choriomeningitis virus (LCMV) glycoprotein, will enable anterograde monosynaptic tracing, offering directional choice [53]. This method is promising and powerful not only for investigating synaptic connectivity and performing physiological characterizations, but, when implemented with recombinant viruses expressing activity indicators (e.g., GCaMP), can also be used to monitor neuronal activity in specific circuits [40]. To date, however, these genetic products are sometimes fairly toxic, and trans-synaptic jumps can be biased by cell type leading to incomplete (40-85\%) presynaptic labeling [49], thus limiting the utility of this technique.

New LM: Super-resolution LM and SPIM with transparent brain

Thus far, we have described methods for overcoming the diffraction limit of LM to resolve synapses by genetic multiple coloring (Brainbow), synaptic immunolabeling with multiple markers (AT), and molecular engineering (mGRASP and rabies virus). Recently, super-resolution microscopy based on non-linear optics with nanometer resolution (i.e., SIM [54], PALM [55], STORM [56], STED [57]) has shown the potential to overcome the resolution limit of light. Such direct optical advances are helpful when combined with methods for mapping synaptic connectivity such as Brainbow and AT, but there is still room for improvement in mapping circuits in the large three-dimensional volume of the brain.

For the 3D reconstruction of an intact brain region, a whole mouse brain, or even a living creature, fluorescent selective plane illumination microscopy (fSPIM) along with optically cleared specimens have recently been introduced [58-60]. SPIM uses a thin laminar sheet of light to illuminate the focal plane, unlike confocal microscopy, which uses cone-shape illumination through the specimen and a spatial pinhole to remove out-of-focus rays. In fSPIM, only the focal plane is illuminated, greatly reducing depth-dependent photobleaching (Fig. 1B). In addition, providing illumination orthogonal to the detection axis allows for deep penetration of light independent of the depth of the focal plane for optical sectioning. This technique also alleviates section distortion and eliminates the challenges of image alignment. Very recent improvements to fSPIM include reducing the width of the light sheet and allowing multi-view reconstruction for better resolution and minimal scattering $[61,62]$. Since fSPIM is most 
effective when used with transparent specimens, new optical clearing methods for fluorescence labeled tissues have been developed in parallel [59, 63, 64]. In particular, the recently developed clearing method called CLARITY improves a specimen's imageable depth, and provides further improvements in transparency, the faithful preservation of fluorescent signals, and stabilization of the sample volume [64]. In our view, fSPIM and new clearing methods together with advanced synaptic detection methods (such as mGRASP and trans-synaptic tracers) are very promising developments for the complete mapping of mammalian synaptic connectivity.

\section{Functional connectivity}

It is of critical importance to understand the relationship between the structure of a neuronal network and its function. Both the EM and LM approaches for visualizing connectivity described above provide only structural information about synaptic connectivity. In recent years, optogenetic approaches (e.g., channelrhodopsin) have accelerated mapping the spatial distribution of synaptic connections together with measures of synaptic strength $[65,66]$, yet these techniques can yield ambiguous results owing to the low resolution of opto-stimulation. To overcome this issue, recent studies have focused on a combination of optogenetic approaches and two-photon microscopic calcium imaging that can precisely detect active synapses innervated by different inputs [67, 68]. At the network level, a powerful, albeit technically challenging, way to determine functional connectivity is to first employ calcium imaging to characterize functional properties, then prepare and image the sample using EM to reconstruct the circuit $[69,70]$. One such study demonstrated that a property of connectivity, asymmetry of wiring, contributes to a specific computation-direction selectivity: the dendrites of mouse starburst amacrine cells make highly specific synapses onto direction-selective ganglion cells in ways that depend on the ganglion cell's preferred direction [70]. The relatively low throughput of the EM approach, though, hinders mapping structure and function in large volumes of whole circuits in the mammalian brain. Combinations of LM approaches described above with task-specific optimizations might also be suitable and may make these analyses more practical.

\section{Synaptic connections in neurological disorders}

Although several neurological disorders may be caused by abnormal synaptic connectivity, efforts to understand this mechanism have been impeded by the lack of suitable techniques. Particularly, autism spectrum disorders (ASD) have been suspected to result from surfeits or deficits of synaptic connectivity [71]. ASD is a group of conditions characterized by impaired social interaction and communication, and restrictive and repetitive behaviors. The number of children diagnosed with ASD continues to rise and ADS has become an important social concern. Studies of genome-wide screening of ASD patients point to possible susceptibility genes [72-79], and several studies with genetically manipulated mice are presently investigating the consequences of losing these genes. Intriguingly, studies in these ASD model mice have reported possible alterations in synaptic connectivity in certain brain regions implicated in ASD. Shank3 mutant mice [80] showed decreased spine density in medium spiny neurons (MSNs) of the striatum, while eIF4E-overexpressing transgenic mice [81] showed increased spine density in pyramidal neurons of the medial prefrontal cortex (mPFC). Although these results are consistent with abnormal connectivity, direct evidence for this is lacking, especially with respect to lengthy connections among brain regions. mGRASP technology would appear to offer a useful approach to test ideas about ASD because mGRASP can quickly and accurately detect actual synapses. A clear understanding of connectivity characteristics associated with ASD will guide us to understanding its cause, and to better diagnosis and treatment.

\section{Interactions with computational modeling}

Not only is synaptic reconstruction technically challenging, but analyzing the reconstructed connections is nontrivial as well. Unlike an organism's genome, which is well-defined and relatively stable, connectivity is well known to be variable and dynamic, changing with, for example, experience-related plasticity. Nonetheless, powerful new computational analyses, coupled with newly revealed synaptic connectivity, have the potential to yield significant insights regarding the principles of synaptic connectivity. First, at the most ambitious level, if the full set of connections between all neurons in a network can be mapped (and single unit dynamics are known) then the dynamics of the full circuit can, at least in principle, be calculated [18]. Such an achievement could yield great insight regarding the operation of the circuit, for example the ability to compute the output associated with each input (though this would require additionally knowing the sign and weight of each mapped connection). Of course, even partial synaptic reconstructions could help constrain the assumptions made when developing models of neural circuits. For instance, existence of highly connected clusters can have a significant effect on a circuit's 
Table 1 Summarized advantages and limitations of synaptic mapping methods

\begin{tabular}{|c|c|c|c|}
\hline Approach & Methods & Advantages & Limitations \\
\hline EM-based & $\begin{array}{l}\text { SBFEM [22, 23], SSSEM with } \\
\text { ATLUM [24], and ATEM [25] }\end{array}$ & $\begin{array}{l}\text { High voxel resolution to reconstruct the synaptic } \\
\text { connectivity of all kinds of cells in dense neural } \\
\text { circuits } \\
\text { Complete picture of neuronal structure }\end{array}$ & $\begin{array}{l}\text { Extremely time- and labor-intensive } \\
\text { Volume-limited }\end{array}$ \\
\hline \multirow[t]{4}{*}{ LM-based } & Brainbow $[8,31]$ & $\begin{array}{l}\text { Dense reconstruction of multi-colored neurons } \\
\text { using LM } \\
\text { Ease and high speed of projection-pattern recon- } \\
\text { struction } \\
\text { Time-lapse imaging or developmental studies }\end{array}$ & $\begin{array}{l}\text { Ambiguous detection of actual } \\
\text { synapse }\end{array}$ \\
\hline & Array tomography $[35,36]$ & $\begin{array}{l}\text { Improved z-resolution } \\
\text { Reliable reconstruction and quantitative analysis } \\
\text { of neural circuits in large volumes of tissue. } \\
\text { EM compatibility } \\
\text { Insights into single-synapse proteome }\end{array}$ & $\begin{array}{l}\text { Decrease of antigenicity and fidelity } \\
\text { of costly antibodies }\end{array}$ \\
\hline & mGRASP $[37,38]$ & $\begin{array}{l}\text { Accurate nanometer-scale detection of synapses } \\
\text { with LM } \\
\text { Quick and suitable for imaging large-scale con- } \\
\text { nectivity patterns }\end{array}$ & $\begin{array}{l}\text { Obscures absolute number of syn- } \\
\text { apses due to false negatives } \\
\text { Limited to pairs }\end{array}$ \\
\hline & $\begin{array}{l}\text { Trans-synaptic tracing by rabies } \\
\text { virus }[41,51]\end{array}$ & $\begin{array}{l}\text { Elucidation of unknown mono-synaptic connec- } \\
\text { tivity throughout the brain } \\
\text { Combinational possibility with neuronal activity } \\
\text { monitoring and manipulating (e.g., GCaMP, } \\
\text { ChR2) }\end{array}$ & $\begin{array}{l}\text { Cytotoxicity of rabies infection } \\
\text { Relatively highly biased to false } \\
\text { negatives }\end{array}$ \\
\hline
\end{tabular}

dynamics [82] and would be difficult to directly ascertain without larger-scale synaptic mapping. Second, the functions of an unidentified neuron could be inferred computationally from the functions of known neurons connected to it. For example, the types of sensory input a neuron processes may be inferred from its connections to identified receptors, or the stimulus preference of a neuron may be deduced from the biases of its input neurons [70]. Third, the architecture of a circuit may provide clues about its computational function. For instance, if a circuit is found to have widespread lateral inhibitory connections, it may be implementing a "winner-takes-all" single-option selection computation whereby the selection of a maximally excited feature is sharpened by the inhibition of the rest of the network [83]. Fourth, analysis of the connectivity may allow us to discover substructures within a given network, revealing an important level of order in the network [84, 85]. Finally, since computational neuroscientists had, until recently, only very few synaptic reconstructions to work from, it is very likely that the novel scope and nature of the data revealed by modern synaptic mapping will inspire new computational methods to reveal conserved properties across different connectomes, and to test the relation between structure and function. In our view, mapping synaptic connectivity is likely to reveal discrete and repeating "modules" within a circuit, providing a level of description between the single neuron micro-level to the macro-level of the circuit as a whole. Such a finding would be immensely important as we strive toward a deeper understanding of neural circuits.

\section{Conclusions and perspectives}

We have reviewed techniques currently available for imaging mammalian synaptic connectivity (Table 1). Unfortunately, thus far, none of these techniques is perfect. None, for example, assesses synaptic strength and efficacy. Innovative new technologies are still required. Meanwhile, creative combinations of all the above techniques, possibly including functional assessments, will go a long way toward allowing mapping of the brain. Stochastic multicolor labeling of Brainbow combined with mGRASP, for instance, could identify the presynaptic partners of a given neuron; it would require labeling each neuron and preparing dense reconstructions of synaptic connectivity under LM. EM combined with new versions of Brainbowexpressing antigenically distinct fluorescent proteins might expand dense reconstructions to encompass long-range connectivity [32]. Also, mGRASP combined with a new retrograde label virus [86, 87] system could help unlock the secrets of disynaptic circuits as well as monosynaptic pairs of cells, and the common drawback of all methods for anatomical synaptic mapping, a lack of information about synaptic activity and strength, can be overcome through combinations of techniques including existing activity indicators and optogenetic tools. 
Open Access This article is distributed under the terms of the Creative Commons Attribution License which permits any use, distribution, and reproduction in any medium, provided the original author(s) and the source are credited.

\section{References}

1. Sotelo C (2003) Viewing the brain through the master hand of Ramón y Cajal. Nat Rev Neurosci 4:71-77

2. Llinás RR (2003) The contribution of Santiago Ramón y Cajal to functional neuroscience. Nat Rev Neurosci 4:77-80

3. Markram H, Lubke J, Frotscher M, Roth A, Sakmann B (1997) Physiology and anatomy of synaptic connections between thick tufted pyramidal neurones in the developing rat neocortex. $\mathrm{J}$ Physiol 500(Pt 2):409-440

4. Kleinfeld D, Bharioke A, Blinder P, Bock DD, Briggman KL, Chklovskii DB, Denk W, Helmstaedter M, Kaufhold JP, Lee WC, Meyer HS et al (2011) Large-scale automated histology in the pursuit of connectomes. J Neurosci: Off J Soc Neurosci 31(45):16125-16138

5. Smith SJ (2007) Circuit reconstruction tools today. Curr Opin Neurobiol 17:601-608

6. Isacoff E, Smith SJ (2009) New technologies. Curr Opin Neurobiol 19:511-512

7. Lichtman JW, Livet J, Sanes JR (2008) A technicolour approach to the connectome. Nat Rev Neurosci 9(6):417-422

8. Lichtman JW, Sanes JR (2008) Ome sweet ome: what can the genome tell us about the connectome? Curr Opin Neurobiol 18:346-353

9. Behrens TEJ, Sporns O (2012) Human connectomics. Curr Opin Neurobiol 22:144-153

10. Wickersham IR, Feinberg EH (2012) New technologies for imaging synaptic partners. Curr Opin Neurobiol 22:121-127

11. Sporns O, Tononi G, Kotter R (2005) The human connectome: a structural description of the human brain. PLoS Comput Biol 1(4):e42

12. Sporns O (2011) The human connectome: a complex network. Ann N Y Acad Sci 1224:109-125

13. Van Essen DC, Ugurbil K (2012) The future of the human connectome. Neuroimage 62:1299-1310

14. White JG, Southgate E, Thomson JN, Brenner S (1986) The structure of the nervous system of the nematode Caenorhabditis elegans. Philos Trans R Soc Lond B Biol Sci 314(1165):1-340

15. Chklovskii DB, Vitaladevuni S, Scheffer LK (2010) Semi-automated reconstruction of neural circuits using electron microscopy. Curr Opin Neurobiol 20(5):667-675

16. Nature Neuroscience Editorial (2010) A critical look at connectomics. Nat Neurosci 13:1441

17. The Washington University Genome Sequencing Center (1998) Genome sequence of the nematode $C$. elegans: a platform for investigating biology. Science 282(5396):2012-2018

18. Seung HS (2009) Reading the book of memory: sparse sampling versus dense mapping of connectomes. Neuron 62:17-29

19. Pastrana E (2013) Focus on mapping the brain. Nat Methods 10(6):481

20. Cajal SR (1888) Estructura de los centros nerviosos de las aves. Rev Trim Histol Norm Patol 1(1):1-10

21. DeFelipe J (2010) From the connectome to the synaptome: an epic love story. Science 330:1198-1201

22. Denk W, Horstmann H (2004) Serial block-face scanning electron microscopy to reconstruct three-dimensional tissue nanostructure. PLoS Biol 2(11):e329

23. Knott G, Marchman H, Wall D, Lich B (2008) Serial section scanning electron microscopy of adult brain tissue using focused ion beam milling. J Neurosci: Off J Soc Neurosci 28(12):2959-2964
24. Helmstaedter M, Briggman KL, Denk W (2008) 3D structural imaging of the brain with photons and electrons. Curr Opin Neurobiol 18:633-641

25. Anderson JR, Jones BW, Yang JH, Shaw MV, Watt CB, Koshevoy P, Spaltenstein J, Jurrus E, UV K, Whitaker RT, Mastronarde D et al (2009) A computational framework for ultrastructural mapping of neural circuitry. PLoS Biol 7(3):e1000074

26. Markram H, Lübke J, Frotscher M, Roth A, Sakmann B (1997) Physiology and anatomy of synaptic connections between thick tufted pyramidal neurones in the developing rat neocortex. J Physiol 500(Pt 2):409-440

27. Lübke J, Roth A, Feldmeyer D, Sakmann B (2003) Morphometric analysis of the columnar innervation domain of neurons connecting layer 4 and layer $2 / 3$ of juvenile rat barrel cortex. Cereb Cortex (New York, NY: 1991) 13(10):1051-1063

28. Andres B, Köthe U, Helmstaedter M, Denk W, Hamprecht FA (2008) Segmentation of SBFSEM volume data of neural tissue by hierarchical classification. In: Pattern recognition. Lecture notes in computer science, vol 5096. Springer, Berlin, pp 142-152

29. Jain V, Murray JF, Roth F, Turaga S, Zhigulin V, Briggman KL, Helmstaedter MN, Denk W, Seung HS (2007) Supervised learning of image restoration with convolution networks. In: Proceedings of the IEEE international conference on computer vision (ICCV)

30. Gan WB, Grutzendler J, Wong WT, Wong RO, Lichtman JW (2000) Multicolor "diolistic" labeling of the nervous system using lipophilic dye combinations. Neuron 27:219-225

31. Livet J, Weissman TA, Kang H, Draft RW, Lu J, Bennis RA, Sanes JR, Lichtman JW (2007) Transgenic strategies for combinatorial expression of fluorescent proteins in the nervous system. Nature 450:56-62

32. Cai D, Cohen KB, Luo T, Lichtman JW, Sanes JR (2013) Improved tools for the Brainbow toolbox. Nat Methods 10(6): 540-547

33. Lu J, Fiala JC, Lichtman JW (2009) Semi-automated reconstruction of neural processes from large numbers of fluorescence images. PLoS One 4:e5655

34. Lu J, Tapia JC, White OL, Lichtman JW (2009) The interscutularis muscle connectome. PLoS Biol 7:e32

35. Micheva KD, Smith SJ (2007) Array tomography: a new tool for imaging the molecular architecture and ultrastructure of neural circuits. Neuron 55:25-36

36. Micheva KD, Busse B, Weiler NC, O'Rourke N, Smith SJ (2010) Single-synapse analysis of a diverse synapse population: proteomic imaging methods and markers. Neuron 68:639-653

37. Feinberg EH, Vanhoven MK, Bendesky A, Wang G, Fetter RD, Shen K, Bargmann CI (2008) GFP reconstitution across synaptic partners (GRASP) defines cell contacts and synapses in living nervous systems. Neuron 57:353-363

38. Kim J, Zhao T, Petralia RS, Yu Y, Peng H, Myers E, Magee JC (2012) mGRASP enables mapping mammalian synaptic connectivity with light microscopy. Nat Methods 9(1):96-102

39. Feng L, Zhao T, Kim J (2012) Improved synapse detection for mGRASP-assisted brain connectivity. Bioinformatics 28: i25-i31

40. Osakada F, Mori T, Cetin AH, Marshel JH, Virgen B, Callaway EM (2011) New rabies virus variants for monitoring and manipulating activity and gene expression in defined neural circuits. Neuron 71:617-631

41. Wickersham IR, Finke S, Conzelmann K-K, Callaway EM (2007) Retrograde neuronal tracing with a deletion-mutant rabies virus. Nat Methods 4(1):47-49

42. Wickersham IR, Lyon DC, Barnard RJO, Mori T, Finke S, Conzelmann K-K, Young JAT, Callaway EM (2007) Monosynaptic restriction of transsynaptic tracing from single, genetically targeted neurons. Neuron 53(5):639-647 
43. Conzelmann KK, Cox JH, Schneider LG, Thiel HJ (1990) Molecular cloning and complete nucleotide sequence of the attenuated rabies virus SAD B19. Virology 175(2):485-499

44. Fields BN, Knipe DM, Howley PM (2007) Fields virology, 5e. Lippincott Williams \& Wilkins, Philadelphia

45. Lafon M (2005) Rabies virus receptors. J Neurovirol 11(1):82-87

46. Ugolini G (2008) Use of rabies virus as a transneuronal tracer of neuronal connections: implications for the understanding of rabies pathogenesis. Dev Biol 131:493-506

47. Ugolini G (1995) Specificity of rabies virus as a transneuronal tracer of motor networks: transfer from hypoglossal motoneurons to connected second-order and higher order central nervous system cell groups. J Comp Neurol 356(3):457-480

48. Kelly RM, Strick PL (2000) Rabies as a transneuronal tracer of circuits in the central nervous system. J Neurosci Methods 103(1):63-71

49. Miyamichi K, Amat F, Moussavi F, Wang C, Wickersham I, Wall NR, Taniguchi H, Tasic B, Huang ZJ, He Z, Callaway EM et al (2011) Cortical representations of olfactory input by trans-synaptic tracing. Nature 472(7342):191-196

50. Ugolini G (1995) Specificity of rabies virus as a transneuronal tracer of motor networks: transfer from hypoglossal motoneurons to connected second-order and higher order central nervous system cell groups. J Comp Neurol 356(3):457-480

51. Callaway EM (2008) Transneuronal circuit tracing with neurotropic viruses. Curr Opin Neurobiol 18:617-623

52. Marshel JH, Mori T, Nielsen KJ, Callaway EM (2010) Targeting single neuronal networks for gene expression and cell labeling in vivo. Neuron 67:562-574

53. Beier KT, Saunders A, Oldenburg IA, Miyamichi K, Akhtar N, Luo L, Whelan SP, Sabatini B, Cepko CL (2011) Anterograde or retrograde transsynaptic labeling of CNS neurons with vesicular stomatitis virus vectors. Proc Natl Acad Sci USA 108(37):15414-15419

54. Gustafsson MGL (2005) Nonlinear structured-illumination microscopy: wide-field fluorescence imaging with theoretically unlimited resolution. Proc Natl Acad Sci USA 102(37):13081-13086

55. Betzig E, Patterson GH, Sougrat R, Lindwasser OW, Olenych S, Bonifacino JS, Davidson MW, Lippincott-Schwartz J, Hess HF (2006) Imaging intracellular fluorescent proteins at nanometer resolution. Science 313:1642-1645

56. Rust MJ, Bates M, Zhuang X (2006) Sub-diffraction-limit imaging by stochastic optical reconstruction microscopy (STORM). Nat Methods 3(10):793-795

57. Willig KI, Rizzoli SO, Westphal V, Jahn R, Hell SW (2006) STED microscopy reveals that synaptotagmin remains clustered after synaptic vesicle exocytosis. Nature 440(7086):935-939

58. Dodt H-U, Leischner U, Schierloh A, Jährling N, Mauch CP, Deininger K, Deussing JM, Eder M, Zieglgänsberger W, Becker K (2007) Ultramicroscopy: three-dimensional visualization of neuronal networks in the whole mouse brain. Nat Methods 4:331-336

59. Mertz J, Kim J (2010) Scanning light-sheet microscopy in the whole mouse brain with hilo background rejection. J Biomed Opt 15:016027

60. Keller PJ, Schmidt AD, Santella A, Khairy K, Bao Z, Wittbrodt J, Stelzer EHK (2010) Fast, high-contrast imaging of animal development with scanned light sheet-based structured-illumination microscopy. Nat Methods 7:637-642

61. Tomer R, Khairy K, Amat F, Keller PJ (2012) Quantitative highspeed imaging of entire developing embryos with simultaneous multiview light-sheet microscopy. Nat Methods 9:755-763

62. York AG, Parekh SH, Dalle Nogare D, Fischer RS, Temprine K, Mione M, Chitnis AB, Combs CA, Shroff H (2012) Resolution doubling in live, multicellular organisms via multifocal structured illumination microscopy. Nat Methods 9(7):749-754

63. Hama H, Kurokawa H, Kawano H, Ando R, Shimogori T, Noda H, Fukami K, Sakaue-Sawano A, Miyawaki A (2011) Scale: a chemical approach for fluorescence imaging and reconstruction of transparent mouse brain. Nat Neurosci 14(11):1481-1488

64. Chung K, Wallace J, Kim SY, Kalyanasundaram S, Andalman AS, Davidson TJ, Mirzabekov JJ, Zalocusky KA, Mattis J, Denisin AK, Pak S et al (2013) Structural and molecular interrogation of intact biological systems. Nature 497(7449):332-337

65. Petreanu L, Huber D, Sobczyk A, Svoboda K (2007) Channelrhodopsin-2-assisted circuit mapping of long-range callosal projections. Nat Neurosci 10:663-668

66. Wang H, Peca J, Matsuzaki M, Matsuzaki K, Noguchi J, Qiu L, Wang D, Zhang F, Boyden E, Deisseroth K, Kasai H et al (2007) High-speed mapping of synaptic connectivity using photostimulation in channelrhodopsin-2 transgenic mice. Proc Natl Acad Sci USA 104:8143-8148

67. Little JP, Carter AG (2012) Subcellular synaptic connectivity of layer 2 pyramidal neurons in the medial prefrontal cortex. J Neurosci: Off J Soc Neurosci 32(37):12808-12819

68. MacAskill AF, Little JP, Cassel JM, Carter AG (2012) Subcellular connectivity underlies pathway-specific signaling in the nucleus accumbens. Nat Neurosci 15(12):1624-1626

69. Bock DD, Lee W-CA, Kerlin AM, Andermann ML, Hood G, Wetzel AW, Yurgenson S, Soucy ER, Kim HS, Reid RC (2011) Network anatomy and in vivo physiology of visual cortical neurons. Nature 471:177-182

70. Briggman KL, Helmstaedter M, Denk W (2011) Wiring specificity in the direction-selectivity circuit of the retina. Nature 471:183-188

71. Belmonte MK, Allen G, Beckel-Mitchener A, Boulanger LM, Carper RA, Webb SJ (2004) Autism and abnormal development of brain connectivity. J Neurosci 24:9228-9231

72. Abrahams BS, Geschwind DH (2008) Advances in autism genetics: On the threshold of a new neurobiology. Nat Rev Genet 9:341-355

73. Marshall CR, Noor A, Vincent JB, Lionel AC, Feuk L, Skaug J, Shago M, Moessner R, Pinto D, Ren Y, Thiruvahindrapduram B et al (2008) Structural variation of chromosomes in autism spectrum disorder. Am J Hum Genet 82:477-488

74. Glessner JT, Wang K, Cai G, Korvatska O, Kim CE, Wood S, Zhang H, Estes A, Brune CW, Bradfield JP, Imielinski M et al (2009) Autism genome-wide copy number variation reveals ubiquitin and neuronal genes. Nature 459:569-573

75. Bucan M, Abrahams BS, Wang K, Glessner JT, Herman EI, Sonnenblick LI, Alvarez Retuerto AI, Imielinski M, Hadley D, Bradfield JP, Kim C et al (2009) Genome-wide analyses of exonic copy number variants in a family-based study point to novel autism susceptibility genes. PLoS Genet 5:e1000536

76. Pinto D, Pagnamenta AT, Klei L, Anney R, Merico D, Regan R, Conroy J, Magalhaes TR, Correia C, Abrahams BS, Almeida J et al (2010) Functional impact of global rare copy number variation in autism spectrum disorders. Nature 466:368-372

77. O'Roak BJ, Vives L, Girirajan S, Karakoc E, Krumm N, Coe BP, Levy R, Ko A, Lee C, Smith JD, Turner EH et al (2012) Sporadic autism exomes reveal a highly interconnected protein network of de novo mutations. Nature 485(7397):246-250

78. Sanders SJ, Murtha MT, Gupta AR, Murdoch JD, Raubeson MJ, Willsey AJ, Ercan-Sencicek AG, DiLullo NM, Parikshak NN, Stein JL, Walker MF et al (2012) De novo mutations revealed by whole-exome sequencing are strongly associated with autism. Nature 485:237-241

79. Neale BM, Kou Y, Liu L, Ma'ayan A, Samocha KE, Sabo A, Lin C-F, Stevens C, Wang L-S, Makarov V, Polak P et al (2012) 
Patterns and rates of exonic de novo mutations in autism spectrum disorders. Nature 485:242-245

80. Peça J, Feliciano C, Ting JT, Wang W, Wells MF, Venkatraman TN, Lascola CD, Fu Z, Feng G (2011) Shank3 mutant mice display autistic-like behaviours and striatal dysfunction. Nature 472:437-442

81. Santini E, Huynh TN, MacAskill AF, Carter AG, Pierre P, Ruggero D, Kaphzan H, Klann E (2013) Exaggerated translation causes synaptic and behavioural aberrations associated with autism. Nature 493:411-415

82. Litwin-Kumar A, Doiron B (2012) Slow dynamics and high variability in balanced cortical networks with clustered connections. Nat Neurosci 15(11):1498-1505

83. Kurt S, Deutscher A, Crook JM, Ohl FW, Budinger E, Moeller CK, Scheich H, Schulze H (2008) Auditory cortical contrast enhancing by global winner-take-all inhibitory interactions. PLoS One 3(3):e1735
84. Song S, Sjostrom PJ, Reigl M, Nelson S, Chklovskii DB (2005) Highly nonrandom features of synaptic connectivity in local cortical circuits. PLoS Biol 3(3):e68

85. Perin R, Berger TK, Markram H (2011) A synaptic organizing principle for cortical neuronal groups. Proc Natl Acad Sci USA 108(13):5419-5424

86. Kato S, Kobayashi K, Inoue K, Kuramochi M, Okada T, Yaginuma H, Morimoto K, Shimada T, Takada M, Kobayashi K (2011) A lentiviral strategy for highly efficient retrograde gene transfer by pseudotyping with fusion envelope glycoprotein. Hum Gene Ther 22:197-206

87. Kato S, Kuramochi M, Takasumi K, Kobayashi K, Inoue K, Takahara D, Hitoshi S, Ikenaka K, Shimada T, Takada M, Kobayashi K (2011) Neuron-specific gene transfer through retrograde transport of lentiviral vector pseudotyped with a novel type of fusion envelope glycoprotein. Hum Gene Ther 22:1511-1523 